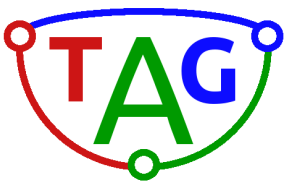

Theory and Applications of Graphs

Volume 6 | Issue 2

Article 5

November 2019

\title{
Conditional Strong Matching Preclusion of the Alternating Group Graph
}

\author{
Mohamad Abdallah \\ American University of Kuwait, mkmabdall@gmail.com \\ Eddie Cheng \\ Oakland University, echeng@oakland.edu
}

Follow this and additional works at: https://digitalcommons.georgiasouthern.edu/tag

\section{Recommended Citation}

Abdallah, Mohamad and Cheng, Eddie (2019) "Conditional Strong Matching Preclusion of the Alternating Group Graph," Theory and Applications of Graphs: Vol. 6 : Iss. 2 , Article 5.

DOI: 10.20429/tag.2019.060205

Available at: https://digitalcommons.georgiasouthern.edu/tag/vol6/iss2/5

This article is brought to you for free and open access by the Journals at Digital Commons@Georgia Southern. It has been accepted for inclusion in Theory and Applications of Graphs by an authorized administrator of Digital Commons@Georgia Southern. For more information, please contact digitalcommons@georgiasouthern.edu. 


\section{Conditional Strong Matching Preclusion of the Alternating Group Graph}

\section{Cover Page Footnote}

We would like to thank the anonymous referees for a number of helpful comments and suggestions. 
Abdallah and Cheng: Strong M atching Preclusion of Alternating Group Graph

\begin{abstract}
The strong matching preclusion number of a graph is the minimum number of vertices and edges whose deletion results in a graph that has neither perfect matchings nor almost-perfect matchings. Park and Ihm introduced the problem of strong matching preclusion under the condition that no isolated vertex is created as a result of faults. In this paper, we find the conditional strong matching preclusion number for the $n$-dimensional alternating group graph $A G_{n}$.
\end{abstract}

\title{
1 Introduction
}

Given a graph $G=(V, E)$, a set $M$ of pairwise nonadjacent edges is called matching. A perfect matching $M$ in $G$ is a matching such that every vertex in $G$ is incident to exactly one edge in $M$. An almost-perfect matching in $G$ is a set of edges such that every vertex in $G$, except one, is incident with exactly one edge in $M$, and the exceptional vertex is incident to none. If $G$ has a perfect matching, then $G$ has an even number of vertices; if $G$ has an almost-perfect matching, then $G$ has an odd number of vertices. We say that the graph $G$ is matchable if it has either a perfect matching or an almost-perfect matching. Otherwise, it is called unmatchable.

A matching preclusion set of $G$ is a set of edges whose deletion results in an unmatchable graph [3]. The matching preclusion number of $G$, denoted by $m p(G)$, is the minimum size of all possible matching preclusion sets of $G$. Any such optimal set is called an optimal matching preclusion set. If $G$ is unmatchable, then $m p(G)=0$. Brigham et al. [3] introduced the concept of matching preclusion as a measure of robustness in the event of edge failure in interconnection networks, as well as a theoretical connection to conditional connectivity.

A trivial case of matching preclusion occurs when all edges in $G$ incident to a single vertex are deleted when $G$ has even number of vertices, or when all edges in $G$ incident to two particular vertices are deleted when $G$ has an odd number of vertices. This case models a situation where link failures are concentrated at only a very few nodes of a communication network. When such case is unlikely to happen, Cheng et al. [10] introduced a useful notion called conditional matching preclusion which removes from consideration the case when the matching preclusion set produces a graph with an isolated vertex after the edge deletion. The conditional matching preclusion number, denoted $m p_{1}(G)$, is the minimum size of all conditional matching preclusion sets of $G$.

Park and Ihm [7] introduced the concept of strong matching preclusion where the matching preclusion set can contain vertices in additional to edges. This concept corresponds to the type of failure in a communication network which occurs through nodes and communication lines. The strong matching preclusion set of $G$ is a set of vertices and/or edges whose deletion leads to an unmatchable graph. The strong matching preclusion number is the minimum size of strong matching preclusion sets in $G$. For the same reason Cheng et al. introduced the conditional matching preclusion, Park and Ihm [8] introduced the concept of conditional strong matching preclusion and discussed its fundamental properties for some classes of graphs and interconnection networks.

A popular class of interconnection networks is the class of alternating group graphs [5]. In this paper we find the conditional strong matching preclusion number of the alternating 
group graph $A G_{n}$, which is the minimum size of all conditional strong matching preclusion sets of $A G_{n}$.

\section{Preliminaries}

A trivial case of matching preclusion occurs when all edges in $G$ incident to a single vertex are deleted when $G$ has an even number of vertices, or when all edges in $G$ incident to two particular vertices are deleted when $G$ has an odd number of vertices. In this paper, our graphs will always have an even number of vertices. We call an optimal solution of the trivial case of matching preclusion a trivial optimal matching preclusion set. Let $F$ be an optimal strong matching preclusion set of a graph $G=(V, E)$, and let $F=F^{V} \cup F^{E}$ where $F^{V}$ consists of vertices in $F$ and $F^{E}$ consists of edges in $F$. We may assume that no element in $F^{E}$ is incident to an element of $F^{V}$ since $F$ is optimal. In fact, if $f \in F^{E}$ is incident to $u \in F^{V}$, then $G-F=G-(F-\{f\})$. If $F$ is an optimal strong matching preclusion set of $G$ and $G-F$ has an isolated vertex, then $F$ is a basic optimal strong matching preclusion set. Based on this definition, it is possible to have a basic optimal matching preclusion set $F$ with $G-F$ odd and without almost-perfect matchings. We can further restrict this class by requiring that, in addition, $G-F$ must be even. Then $F$ is called optimal strong matching preclusion set.

The following proposition considers the relationship between basic strong matching preclusion sets and trivial strong matching preclusion sets.

Proposition 2.1. [4] Let $G$ be a $r$-regular even graph with $r \geq 2$. Suppose that $\operatorname{smp}(G)=r$. Then every basic optimal strong matching preclusion set is trivial.

Let $F \subseteq V(G) \cup E(G), F$ is a conditional strong matching preclusion set of $G$ if $G-F$ has neither a perfect matching nor an almost-perfect matching and no isolated vertices. The minimum cardinality of all such sets is denoted by $\operatorname{smp}_{1}(G)$, and called the conditional strong matching preclusion number of $G$. In this paper, we assume $G$ has no isolated vertices. If $G$ is unmatchable, then $s m p_{1}(G)=0$. The following propositions follow directly from the fact that a matching preclusion set is a special case of a strong matching preclusion set consisting of edges only.

Proposition 2.2. [4] Let $G$ be a graph with an even number of vertices. Then smp $(G) \leq$ $m p(G) \leq \delta(G)$, where $\delta(G)$ is the minimum degree of $G$.

Proposition 2.3. [8] For every graph $G$ for which all the four numbers, $m p(G), m p_{1}(G)$, $\operatorname{smp}(G)$, and $\operatorname{smp}_{1}(G)$ are well defined, $\operatorname{smp}(G) \leq s m p_{1}(G) \leq m p_{1}(G)$ and $\operatorname{smp}(G) \leq$ $m p(G) \leq m p_{1}(G)$.

Under the condition of no isolated vertices allowed after the deletion of edges and/or vertices, an easy way to build a conditional strong matching preclusion set in $G$ is to try a fault set $F$ that leaves after deletion a path $(u, z, v)$ made of the three vertices $u, z$ and $v$, where $\operatorname{deg}_{G-F}(u)=\operatorname{deg}_{G-F}(v)=1$. If $G-F$ is even, then the resulting graph becomes unmatchable. Therefore we can build a candidate conditional strong matching preclusion set as follows. Let $N_{G}(\cdot)$ represents the set of neighboring vertices in $G$. Given a path $(u, z, v)$ in a graph $G=(V, E)$, build a fault set, denoted $F_{u z v}$, in such a way that 
1. $F_{u z v}$ contains every vertex $w \in\left(N_{G}(u) \cap N_{G}(v)\right)-\{z\}$,

2. $F_{u z v}$ contains the edge $u v$ if $u v \in E(G)$,

3. for every vertex $w \in N_{G}(u)-N_{G}(v), F_{u z v}$ contains exactly one of $w$ and $u w$,

4. for every vertex $w \in N_{G}(v)-N_{G}(u), F_{u z v}$ contains exactly one of $w$ and $v w$.

The next fundamental proposition provides sufficient conditions to make $F_{u z v}$ a conditional strong matching preclusion set.

Proposition 2.4. [8] For an arbitrary path $(u, z, v)$ in a graph $G, F_{u z v}$ is a conditional strong matching preclusion set of $G$ if

1. there is no isolated vertex in $G-F_{u z v}$, and

2. $G-F_{u z v}$ has an even number of vertices.

The conditional strong matching preclusion set described in Proposition 2.4 is called trivial as it is one of the simplest ways of building a conditional strong matching preclusion set. The following proposition provides an upper bound for $s m p_{1}(G)$.

Proposition 2.5. [8] If there exists a trivial conditional strong matching preclusion set $F_{u z v}$ for some path $(u, z, v)$ in a graph $G$, then $\operatorname{smp}_{1}(G) \leq \operatorname{deg}_{G}(u)+\operatorname{deg}_{G}(v)-2-g_{G}(u, v)$, where $g_{G}(u, v)$ is $|N(u) \cap N(v)|$ if $(u, v) \in E(G)$ or $|N(u) \cap N(v)|-1$ otherwise.

The alternating group graph was introduced by Jwo et al. [5] as an interconnection network topology for computing systems. Let $A_{n}$ be the alternating group, that is, $A_{n}$ is the set of even permutations of the set $\{1,2, \ldots, n\}$. By [1], the size of $A_{n}$ is $\frac{n !}{2}$ and the set

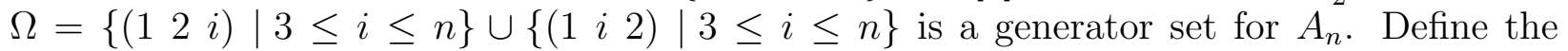
alternating group graph $A G_{n}=\left(V_{n}, E_{n}\right)$ of dimension $n$ as follows: $V_{n}=A_{n}$, the set of all even permutations, and $E_{n}=\left\{(p, q) \mid p, q \in A_{n}, q=p \cdot h\right.$, for $\left.h \in \Omega\right\}$, where "." is the usual binary associative operator defined by $u \cdot v(x)=u(v(x))$. $A G_{n}$ is a Cayley graph of the alternating group [6].

$A G_{n}$ can be recursively built by using $n$ copies of $A G_{n-1}$. Let $H_{i}$ denote the induced subgraph of $A G_{n}$ corresponding to the permutations $p \in A_{n}$ such that the last symbol of $p$ is $i$. Note that instead of last position, we can consider $H_{i}$ according to the $j$ th position for any $3 \leq j \leq n$. We refer to this as a decomposition along the $j$ th position. The following proposition is an easy and known result of the recursive structure of $A G_{n}$ :

Proposition 2.6. Let $A G_{n}$ be the alternating group graph of dimension $n \geq 4$.

(I) $\left|V_{n}\right|=\frac{n !}{2}$ and $\left|E_{n}\right|=\frac{(n-2) n !}{2}$.

(II) $A G_{n}$ is $(2 n-4)$-regular.

(III) $A G_{n}$ consists of $n$ vertex-disjoint subgraphs, $H_{1}, H_{2}, \ldots, H_{n}$, each isomorphic to $A G_{n-1}$.

(IV) $H_{i}$ has $(n-1) ! / 2$ vertices, and it is $(2 n-6)$-regular for all $i$. 
(V) There are exactly $(n-2)$ ! independent edges between $H_{i}$ and $H_{j}$ for all $i \neq j$. An edge between different $H_{i}$ 's will be called a cross edge.

(VI) Each vertex in $H_{i}$ has exactly two neighbors outside $H_{i}$ (which are called its outside neighbors); these two outside neighbors are in different $H_{k}$ 's, and there is an edge between them. Thus every vertex forms a triangle with its two outside neighbors.

(VII) For every different $i, j$, and $k$, there are exactly $(n-3)$ ! vertices in $H_{i}$ that have an outside neighbor in $H_{j}$ and an outside neighbor in $H_{k}$.

Proposition 2.7. [12] Let $u, v \in V_{n}$. Then $|N(u) \cap N(v)| \leq 2$.

Using the results from Proposition 2.5 and Proposition 2.7, we can establish an upper bound for the size of the conditional strong matching preclusion set of $A G_{n}$.

Proposition 2.8. For $n \geq 4$, $s m p_{1}\left(A G_{n}\right) \leq 4 n-11$.

Lemma 2.1. Suppose that a graph $G$ has an almost-perfect matching that misses a vertex $v$ that is not isolated, then there exists another almost-perfect matching in $G$ that misses a vertex other than $v$.

Proof. Let $M$ be an almost-perfect matching in $G$ that misses the vertex $v \in V(G)$. Since $v$ is not isolated, then $v$ is adjacent to some vertex $u \in V(G)$. The matching $M$ saturates $u$, so let the edge $w u \in M$. Let $M^{\prime}=(M-\{w u\}) \cup\{u v\}, M^{\prime}$ is an almost-perfect matching in $G$ that misses the vertex $w$.

Lemma 2.2. Let $F \subseteq V_{n}$ for $n \geq 5$, such that $|F|=4 n-12$. If $F \subseteq V\left(H_{i}\right)$ for some $i \in\{1, \ldots, n\}$, then we can choose the decomposition via a different position so that at most $4 n-15$ of the vertices in $F$ belong to $H_{j}$ for some $j \in\{1, \ldots, n\}$.

Proof. Without loss of generality we can assume that $F \subseteq V\left(H_{n}\right)$, then all the $4 n-12$ vertices end with $n$. We can look at these vertices as a $(4 n-12) \times n$ array, where each row represents the corresponding permutation of each vertex. The $n$th column contains the number $n$ only. If the $(n-1)$ st column contains at most $(4 n-15)$ numbers of the same number $i$ for some $i \in\{1, \ldots, n-1\}$, then we can decompose along this position. Otherwise, this column contains at least $4 n-14 i$ 's for some $i \in\{1,2, \ldots, n-1\}$. For notational convenience, we can assume that $i=n-1$, so the entries of the $(n-1)$ st column must be the number $(n-1)$ except for at most 2 rows. Repeating the same process, and assuming that we do not find the desired position to decompose along, then when we reach the 3rd column we will have at most $2(n-3)$ rows different from

$$
345 \ldots(n-1) n
$$

so we get at least $(4 n-12)-2(n-3)=2 n-6$ rows of the form

$$
345 \ldots(n-1) n
$$

where the first two positions must be 1 and 2. For $n \geq 5$, the number of such vertices is at least 4 , which is not possible. Then we should be able to find a column that contains no 
more than $4 n-15$ numbers of the same number $i$, and therefore decomposing along the $i$ th position guarantees that at most $4 n-15$ vertices are in the new $H_{i}$.

Lemma 2.3. Let $F \subseteq V_{n} \cup E_{n}$ for $n \geq 5$, such that $|F|=4 n-12$. If $F \subseteq V\left(H_{i}\right) \cup E\left(H_{i}\right)$ for some $i \in\{1, \ldots, n\}$, then we can choose the decomposition via a different position so that at most $4 n-15$ of the elements in $F$ belong to $H_{j}$ for some $j \in\{1, \ldots, n\}$.

Proof. Let $F=F^{V} \cup F^{E}$ be a subset of $H_{i}$ for some $i \in\{1,2, \ldots, n\}$, where $F^{V}$ is the set of vertices in $F$ and $F^{E}$ is the set of edges in $F$. Let $F^{V}=\left\{u_{1}, u_{2}, \ldots, u_{p}\right\}$ and $F^{E}=\left\{x_{1} y_{1}, x_{2} y_{2}, \ldots, x_{q} y_{q}\right\}$. We have $p+q=4 n-12$. Consider the set $L=$ $\left\{u_{1}, u_{2}, \ldots, u_{p}, x_{1}, x_{2}, \ldots, x_{q}\right\}$ in $V\left(H_{1}\right)$. By Proposition 2.2, We can decompose via a different position so that at most $4 n-15$ elements of $L$ belong to $H_{j}$ for some $j \in\{1, \ldots, n\}$. Note that if some $x_{i} \in L$ is outside $H_{j}$ and if $y_{i} \in V\left(H_{j}\right)$ then the faulty edge $x_{i} y_{i}$ will be a cross edge and it is not a fault in $H_{j}$. Therefore we can conclude that at most $4 n-15$ of the elements in $F$ belong to $H_{j}$.

Theorem 2.4. [4] Let $n \geq 4$. Then $\operatorname{smp}\left(A G_{n}\right)=2 n-4$. Moreover every optimal strong matching preclusion set of $A G_{n}$ is trivial.

\section{The Main Result}

Theorem 3.1. Let $n \geq 4$. Then $\operatorname{smp}_{1}\left(A G_{n}\right)=4 n-11$.

Proof. Let $F \subseteq V_{n} \cup E_{n}$ be a set of faults in $A G_{n}$ such that $A G_{n}-F$ has no isolated vertices. $F=F^{V} \cup F^{E}$, where $F^{V}=V_{n} \cap F$ and $F^{E}=E_{n} \cap F$. We want to show that if $|F| \leq 4 n-12$, then $A G_{n}-F$ has a perfect or an almost-perfect matching. Suppose $n=4$. If $|F| \leq 4$ then, by Theorem 2.4, $A G_{4}-F$ has a perfect matching or an almost-perfect matching and every matching preclusion set of size 4 is the set of vertices and/or edges that isolates a vertex. Note that when $n=4,2 n-4=4 n-12=4$, so if we delete 4 faults without leaving an isolated vertex in $A G_{n}-F$, the graph $A G_{n}-F$ must contain a perfect or an almost-perfect matching. Then $s m p_{1}\left(A G_{4}\right) \geq 5$. By Proposition 2.8, $s m p_{1}\left(A G_{4}\right) \leq 5$, then we have $\operatorname{smp}_{1}\left(A G_{4}\right)=5$. Hence, the claim is true for $n=4$.

We proceed by induction on $n$. Suppose that $\operatorname{smp}_{1}\left(A G_{n-1}\right)=4(n-1)-11=4 n-15$, we want to show that $s m p_{1}\left(A G_{n}\right)=4 n-11$. Let $F_{i}=F_{i}^{V} \cup F_{i}^{E}$ be the set of faults in $H_{i}$ for $i=1, \ldots, n$. We consider several cases depending on the faults' distribution in the $H_{i}$ 's.

Case 1. $\left|F_{i}\right| \leq 2 n-7$ for all $i \in\{1, \ldots, n\}$. For $i, j \in\{1, \ldots, n\}$ such that $i \neq j$, there are $(n-2) !-(4 n-12)$ possible cross edges between $H_{i}-F_{i}$ and $H_{j}-F_{j}$. So when $n \geq 6$, there are at least 12 possible cross edges. Since $H_{i}$ is $(2 n-6)$-regular and $\left|F_{i}\right| \leq 2 n-7$, then $H_{i}-F_{i}$ contains no isolated vertices, then by Theorem 2.4 (or by the induction hypothesis) there exists a perfect matching or an almost-perfect matching in every $H_{i}-F_{i}$ for $i=1, \ldots, n$. For notational convenience, assume that $\left|F_{1}^{V}\right|, \ldots,\left|F_{k}^{V}\right|$ are odd for some $k \in\{1, \ldots, n\}$, and $\left|F_{k+1}^{V}\right|, \ldots,\left|F_{n}^{V}\right|$ are even. By the induction hypothesis, there exist almost-perfect matchings $M_{i}$ in $H_{i}-F_{i}$ for $i=1, \ldots, k$ and 
perfect matchings $M_{j}$ in $H_{j}-F_{j}$ for $j=k+1, \ldots, n$. As mentioned above, there are at least 12 cross edges between any $H_{i}-F_{i}$ and $H_{j}-F_{j}$ for $i \neq j$. We want to choose a cross edge $e=u v$ in $A G_{n}-F$ between $H_{i}-F_{i}$ and $H_{j}-F_{j}$ where $u \in V\left(H_{i}-F_{i}\right)$ and $v \in V\left(H_{j}-F_{j}\right)$ so that $H_{i}-\left(F_{i} \cup\{u\}\right)$ and $H_{j}-\left(F_{j} \cup\{v\}\right)$ have no isolated vertices. In every $H_{i}-F_{i}$, there could be at most one such vertex $u$ whose deletion produces an isolated vertex in $H_{i}-\left(F_{i} \cup\{u\}\right)$, then there could be at most two cross edges that cannot be chosen. As a result, we can always find a cross edge $e=x_{i} x_{j}$ between $H_{i}-F_{i}$ and $H_{j}-F_{j}$ such that $H_{i}-\left(F_{i} \cup\left\{x_{i}\right\}\right)$ and $H_{j}-\left(F_{j} \cup\left\{x_{j}\right\}\right)$ have no isolated vertices. We consider two possibilities depending on the parity of $k$.

$k$ is even. For $i=1, \ldots, k-1$, pick cross edges $x_{1} x_{2}, x_{3} x_{4}, \ldots, x_{k-1} x_{k} \in E\left(A G_{n}-F\right)$ as described above, where $x_{i} \in V\left(H_{i}-F_{i}\right)$. Thus $H_{i}-\left(F_{i} \cup\left\{x_{i}\right\}\right)$ contains an even number of vertices and has no isolated vertices. Since $\left|F_{i} \cup\left\{x_{i}\right\}\right| \leq 2 n-6$, then by Theorem 2.4 there exist perfect matchings $M_{i}^{\prime}$ in $H_{i}-\left(F_{i} \cup\left\{x_{i}\right\}\right)$ for $i=1, \ldots, k$. Therefore $\bigcup_{i=1}^{k} M_{i}^{\prime} \cup\left\{x_{1} x_{2}, x_{3} x_{4}, \ldots, x_{k-1} x_{k}\right\} \cup \bigcup_{j=k+1}^{n} M_{j}$ is a perfect matching in $A G_{n}-F$.

$k$ is odd. We use the same argument and notation as above. The set $\bigcup_{i=1}^{k-1} M_{i}^{\prime} \cup$ $\left\{x_{1} x_{2}, x_{3} x_{4}, \ldots, x_{k-2} x_{k-1}\right\} \cup \bigcup_{j=k}^{n} M_{j}$ is an almost-perfect matching in $A G_{n}-F$. See Figure 1.

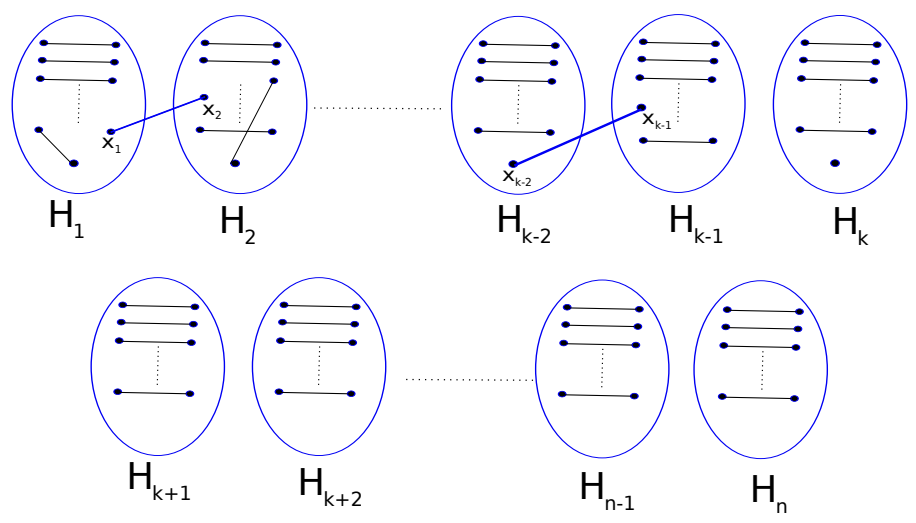

Figure 1: Case 1: $\left|F_{i}\right| \leq 2 n-7$ for $i \in\{1, \ldots, n\}$

Suppose $n=5$. If there is an edge between every pair of $H_{i}-F_{i}$ and $H_{j}-F_{j}$ in $A G_{n}-F$, then we are done. But such a violation can only occur for one pair say $H_{1}-F_{1}$ and $H_{2}-F_{2}$. Thus the graph induced by $V\left(H_{1}\right) \cup V\left(H_{2}\right)$ has at least six faults. So there are only two faults not yet discovered. Now, the above construction works unless $k=2$. (If $k \geq 3$, we can avoid pairing $H_{1}-F_{1}$ and $H_{2}-F_{2}$ ). Since there are only two faults not yet discovered and $n=5$. We may assume that $\left|F_{3}\right|=0$ and none of the cross edges between $H_{1}-F_{1}$ and $H_{3}-F_{3}$, and between $H_{2}-F_{2}$ and $H_{3}-F_{3}$ are faulty edges. Now choose $x_{1} x_{3}$ and $x_{2} x_{3}^{\prime}$ appropriately. Now $H_{3}-\left\{x_{3}, x_{3}^{\prime}\right\}$ has a perfect matching, and we can continue with the usual construction.

Case 2. $\left|F_{1}\right|=2 n-6$ and $\left|F_{i}\right| \leq 2 n-7$ for all $i \neq 1$.

Case 2.1. $H_{1}-F_{1}$ has no isolated vertices. By the induction hypothesis, there exists a perfect matching or an almost-perfect matching $M_{1}$ in $H_{1}-F_{1}$, and by Theorem 2.4 , every 
$H_{i}-F_{i}$ contains a perfect or an almost-perfect matching $M_{i}$ for $i \neq 1$. Then this case is similar to Case 1.

Case 2.2. $H_{1}-F_{1}$ has an isolated vertex $u \in V\left(H_{1}-F_{1}\right)$.

Case 2.2.1. $F_{1}$ is not a matching preclusion set in $H_{1}$. By Proposition $2.1,\left|F_{1}^{V}\right|$ is odd. Then $H_{1}-F_{1}$ has an almost-perfect matching $M_{1}$ missing the vertex $u$. Since $A G_{n}-F$ has no isolated vertices, then the vertex $u$ must be adjacent to some outside neighbor $u^{\prime}$ in $A G_{n}-F$. Without loss of generality, assume that $u^{\prime} \in V\left(H_{2}-F_{2}\right)$. Let $F_{2}^{\prime}=F_{2} \cup\left\{u^{\prime}\right\}$, we consider two cases depending on whether the resulting subgraph induced by $H_{2}-F_{2}^{\prime}$ has an isolated vertex or not.

Case 2.2.1.1. $H_{2}-F_{2}^{\prime}$ has no isolated vertices. Since $\left|F_{2}^{\prime}\right| \leq 2 n-6$, then by the induction hypothesis, $H_{2}-F_{2}^{\prime}$ has a perfect or an almost-perfect matching $M_{2}$. We consider two cases depending on whether $M_{2}$ is a perfect or an almostperfect matching.

$M_{2}$ is a perfect matching. By the induction hypothesis, we can find perfect or almost-perfect matchings $M_{i}$ in $H_{i}-F_{i}$ for $i \geq 3$. Since $\left|F_{i}\right| \leq 2 n-7$ for $i \geq 3$, then we can proceed as in Case 1 and combine those matchings to get a perfect or an almost-perfect matching $M^{\prime}$ in the subgraph induced by $\left(A G_{n}-F\right)-\left(V\left(H_{1}\right) \cup V\left(H_{2}\right)\right)$. Then $M^{\prime} \cup M_{1} \cup M_{2} \cup\left\{u u^{\prime}\right\}$ is a perfect or an almost-perfect matching in $A G_{n}-F$.

$M_{2}$ is an almost-perfect matching. If $\left|F_{2}^{\prime}\right| \leq 2 n-7$, then we can proceed as in Case 1 to find a perfect or an almost-perfect matching $M^{\prime}$ in the subgraph induced by $\left(A G_{n}-F\right)-\left(V\left(H_{1}\right) \cup\left\{u^{\prime}\right\}\right)$, and the set $M^{\prime} \cup$ $M_{1} \cup\left\{u u^{\prime}\right\}$ is a perfect or an almost-perfect matching in $A G_{n}-F$. If $\left|F_{2}^{\prime}\right|=2 n-6$, then let $x \in V\left(H_{2}-F_{2}^{\prime}\right)$ be the unsaturated vertex by $M_{2}$. Under this assumption, there is only one fault left outside the subgraph induced by $V\left(H_{1}\right) \cup V\left(H_{2}\right)$. Since $x$ is not isolated in $H_{2}-F_{2}^{\prime}$, then by Proposition 2.1 there exists another almost-perfect matching in $H_{2}-F_{2}^{\prime}$ missing a vertex different from $x$. So without loss of generality we can assume that $x$ has two outside neighbors in $A G_{n}-F$ or else we consider the other almost-perfect matching. Let $x^{\prime}$ be the outside neighbor of $x$, such that $x^{\prime} \in V\left(H_{j}-F\right)$ for some $j \neq 1,2$. Let $F_{j}^{\prime}=F_{j} \cup\left\{x^{\prime}\right\}$, then by the induction hypothesis there exists a perfect or an almostperfect matching $M_{j}$ in $H_{j}-F_{j}^{\prime}$, and $M_{i}$ in $H_{i}-F_{i}$ for all $i \neq 1,2, j$. Since $\left|F_{j}^{\prime}\right|$ and $\left|F_{i}\right|$ are less than or equal to $2 n-7$, then we can use the construction of Case 1 to find a perfect or an almost-perfect matching $M^{\prime}$ in the subgraph induced by $\left(A G_{n}-F\right)-\left(V\left(H_{1}\right) \cup V\left(H_{2}\right) \cup\left\{u^{\prime}, x^{\prime}\right\}\right)$. Then the set $M^{\prime} \cup M_{1} \cup M_{2} \cup\left\{u u^{\prime}, x x^{\prime}\right\}$ is a perfect or an almost-perfect matching in $A G_{n}-F$.

Case 2.2.1.2. $H_{2}-F_{2}^{\prime}$ has an isolated vertex. Then we can choose the second outside neighbor of $u, u^{\prime \prime}$, which belongs to $V\left(H_{k}\right)$ for some $k \neq 1,2$. If $u^{\prime \prime} \in$ $V\left(H_{k}-F_{k}\right)$ and $u u^{\prime \prime} \in E\left(A G_{n}-F\right)$, then $H_{k}-\left(F_{k} \cup\left\{u^{\prime}\right\}\right)$ has no isolated vertices, and then we can proceed as in the previous case. Assume the worst case scenario when $u^{\prime \prime}$ is a faulty vertex or $u u^{\prime \prime}$ is a faulty edge. We 
have $\left|F_{2}^{\prime}\right|=2 n-6$ and $u^{\prime}$ is adjacent to a vertex $z \in V\left(H_{2}-F_{2}\right)$ and all faults in $H_{2}$ are adjacent and/or incident to $z$ as well. The vertex $z$ has two outside neighbors in $A G_{n}-F$, let $z^{\prime} \in V\left(H_{k}-F_{k}\right)$ be an outside neighbor of $z$ for some $k \neq 1,2$. We consider the parity of $\left|F_{2}^{V}\right|$.

$\left|F_{2}^{V}\right|$ is even. Then there exists a perfect matching $M_{2}$ in $H_{2}-F_{2}$, and in this case the edge $u^{\prime} z$ must belong to $M_{2}$. As in Case 1 , we can find a perfect or an almost-perfect matching $M^{\prime}$ in the subgraph induced by $\left(A G_{n}-F\right)-\left(V\left(H_{1}\right) \cup V\left(H_{2}\right) \cup\left\{z^{\prime}\right\}\right)$. Then $M_{1} \cup\left(M_{2}-\left\{u^{\prime} z\right\}\right) \cup\left\{u u^{\prime}, z z^{\prime}\right\} \cup$ $M^{\prime}$ is a perfect or an almost-perfect matching in $A G_{n}-F$. See Figure 2.

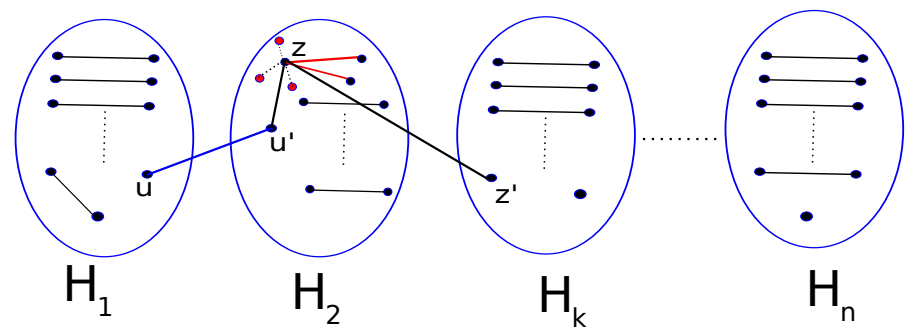

Figure 2: Case 2.2.1: $\left|F_{2}^{V}\right|$ is even

$\left|F_{2}^{V}\right|$ is odd. Then choose an appropriate vertex $x \in V\left(H_{2}-F_{2}\right)$ such that $x$ has an outside neighbor $x^{\prime} \in V\left(H_{p}-F_{p}\right)$ for some $p \neq 1$, 2 with $x x^{\prime} \in E\left(A G_{n}-F\right)$ and $H_{2}-(F \cup\{x\})$ has no isolated vertices. Let $F_{2}^{\prime \prime}=F_{2} \cup\{x\}$, then $\left|F_{2}^{\prime \prime V}\right|$ is even of size $2 n-6$ and has no isolated vertices, then by Theorem $2.4 H_{2}-F_{2}^{\prime \prime}$ has a perfect matching $M_{2}^{\prime}$. By the induction hypothesis, there exists a perfect or an almost-perfect matching $M_{i}$ in $H_{i}-\left(F_{i} \cup\left\{x^{\prime}, z^{\prime}\right\}\right)$ for $i \geq 3$. Since all the faults except one are in the subgraph induced by $V\left(H_{1}\right) \cup V\left(H_{2}\right)$, then $\left|F_{i} \cup\left\{x^{\prime}, z^{\prime}\right\}\right| \leq 2 n-7$ for $i \geq 3$, then we can use the construction of Case 1 to combine the $M_{i}$ 's and get a perfect or an almost-perfect matching $M^{\prime \prime}$ in the subgraph induced by $\left(A G_{n}-F\right)-\left(V\left(H_{1}\right) \cup V\left(H_{2}\right) \cup\left\{x^{\prime}, z^{\prime}\right\}\right)$. Therefore $M_{1} \cup\left(M_{2}^{\prime}-\right.$ $\left.\left\{u^{\prime} z\right\}\right) \cup\left\{u u^{\prime}, z z^{\prime}, x x^{\prime}\right\} \cup M^{\prime \prime}$ is a perfect or an almost-perfect matching in $A G_{n}-F$. See Figure 3 .

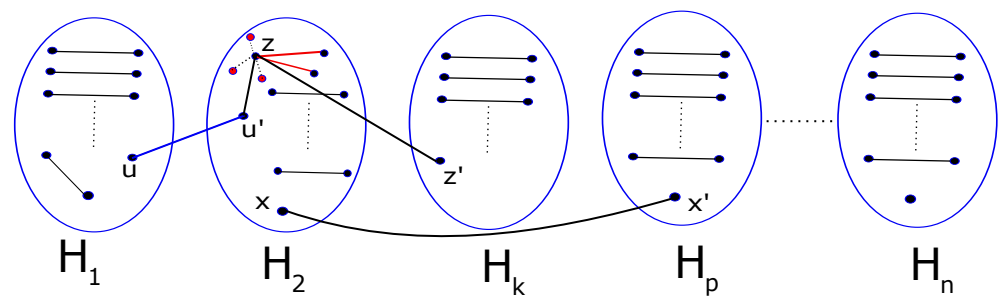

Figure 3: Case 2.2.1: $\left|F_{2}^{V}\right|$ is odd

Case 2.2.2. $F_{1}$ is a matching preclusion set in $H_{1}$. Then $F_{1}$ is a basic strong matching preclusion set. Since $\operatorname{smp}\left(H_{1}\right)=2 n-6$, and $H_{1}$ is even and regular, then by Proposition 2.1 every basic optimal strong matching preclusion set is trivial, therefore $\left|F_{1}^{V}\right|$ is even. 
Case 2.2.2.1. Assume that $F_{1}^{V} \neq \emptyset$. It is easy to find a vertex $w$ in $F_{1}$ such that $H_{1}-\left(F_{1}-\{w\}\right)$ has no isolated vertices. The vertex $w$ must be adjacent to $u$ in $H_{1}-\left(F_{1}-\{w\}\right)$. Since $u$ is isolated in $H_{1}$ and because $A G_{n}-F$ contains no isolated vertices, then $u$ must have an outside neighbor $u^{\prime}$ in $A G_{n}-F$. Without loss of generality, assume that $u^{\prime} \in V\left(H_{2}-F_{2}\right)$. Let $v \in V\left(H_{1}-F_{1}\right)$ such that $H_{1}-\left(F_{1} \cup\{v\}\right)$ has no isolated vertices and $v$ has an outside neighbor $v^{\prime} \in V\left(H_{p}-F_{p}\right)$, with $v v^{\prime} \in E\left(A G_{n}-F\right)$, for some $p \neq 1,2$. Let $F_{1}^{\prime}=\left(F_{1}-\{w\}\right) \cup\{v\}$, then $\left|F_{1}^{\prime}\right|=2 n-6$ and contains an even number of vertices. Moreover, $H_{1}-F_{1}^{\prime}$ has no isolated vertices, then by Theorem 2.4 we can find a perfect matching $M_{1}$ in $H_{1}-F_{1}^{\prime}$. This matching must contain the edge $u w$.

Assume that $\mathrm{H}_{2}-\left(F_{2} \cup\left\{u^{\prime}\right\}\right)$ has no isolated vertices. Then by the induction hypothesis there exists a perfect or an almost-perfect matching $M_{i}$ in $H_{i}-\left(F_{i} \cup\left\{u^{\prime}, v^{\prime}\right\}\right)$ for $i \geq 2$. As in Case 1, we can combine those matchings to get a perfect or an almost-perfect matching $M^{\prime}$ in the subgraph induced by $\left(A G_{n}-F\right)-\left(V\left(H_{1}\right) \cup\left\{u^{\prime}, v^{\prime}\right\}\right)$. Then the set $\left(M_{1}-\{u w\}\right) \cup\left\{u u^{\prime}, v v^{\prime}\right\} \cup M^{\prime}$ is a perfect or an almost-perfect matching in $A G_{n}-F$.

Assume that $\mathrm{H}_{2}-\left(F_{2} \cup\left\{u^{\prime}\right\}\right)$ has an isolated vertex $z$. Under this case we can choose the other outside neighbor of $u, u^{\prime \prime} \in V\left(H_{k}\right)$, for some $k \neq 1,2$. If $u^{\prime \prime}$ is a faulty vertex or $u u^{\prime \prime}$ is a faulty edge, then we can guarantee that the vertex $z$ has two outside neighbors in $A G_{n}-F$. Let $z^{\prime}$ be an outside neighbor of $z$ such that $z^{\prime} \in V\left(H_{k}-F_{k}\right)$ with $z z^{\prime} \in E\left(A G_{n}-F\right)$ for some $k \neq 1,2$.

- If $\left|F_{2}^{V}\right|$ is even, then there exists a perfect matching $M_{2}$ in $H_{2}-F_{2}$, and in this case the edge $u^{\prime} z$ belongs to $M_{2}$. As in Case 1, we can find a perfect or an almost-perfect matching $M^{\prime}$ in the subgraph induced by $\left(A G_{n}-F\right)-\left(V\left(H_{1}\right) \cup V\left(H_{2}\right) \cup\left\{z^{\prime}, v^{\prime}\right\}\right)$. Then $M_{1} \cup\left(M_{2}-\left\{u^{\prime} z\right\}\right) \cup$ $\left\{u u^{\prime}, z z^{\prime}, v v^{\prime}\right\} \cup M^{\prime}$ is a perfect or an almost-perfect matching in $A G_{n}-$ $F$. See Figure 4.

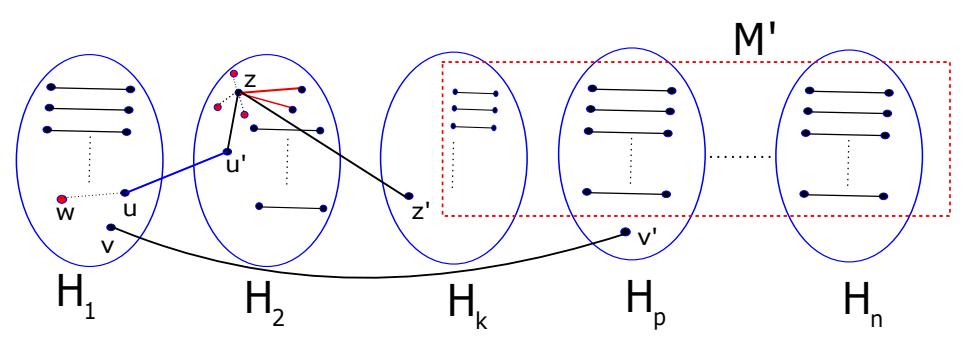

Figure 4: Case 2.2.2.1: $\left|F_{2}^{V}\right|$ is even

- If $\left|F_{2}^{V}\right|$ is odd, then choose a vertex $x \in V\left(H_{2}-F_{2}\right)$ such that $x$ has an outside neighbor $x^{\prime} \in V\left(H_{q}-F_{q}\right)$ for some $q \neq 1,2$ and $H_{2}-\left(F_{2} \cup\{x\}\right)$ has no isolated vertices. Let $F_{2}^{\prime \prime}=F_{2} \cup\{x\}$, then $\left|F_{2}^{\prime \prime V}\right|$ is even and of size $2 n-6$ and has no isolated vertices, then by Theorem $2.4 H_{2}-F_{2}^{\prime \prime}$ has a perfect matching $M_{2}^{\prime}$. As in Case 1, we can find a perfect or an almost- 
perfect matching $M^{\prime \prime}$ in the subgraph induced by $\left(A G_{n}-F\right)-\left(V\left(H_{1}\right) \cup\right.$ $\left.V\left(H_{2}\right) \cup\left\{x^{\prime}, z^{\prime}, v^{\prime}\right\}\right)$. Then $M_{1} \cup\left(M_{2}^{\prime}-\left\{u^{\prime} z\right\}\right) \cup\left\{u u^{\prime}, v v^{\prime}, z z^{\prime}, x x^{\prime}\right\} \cup M^{\prime \prime}$ is a perfect or an almost-perfect matching in $A G_{n}-F$. See Figure 5.

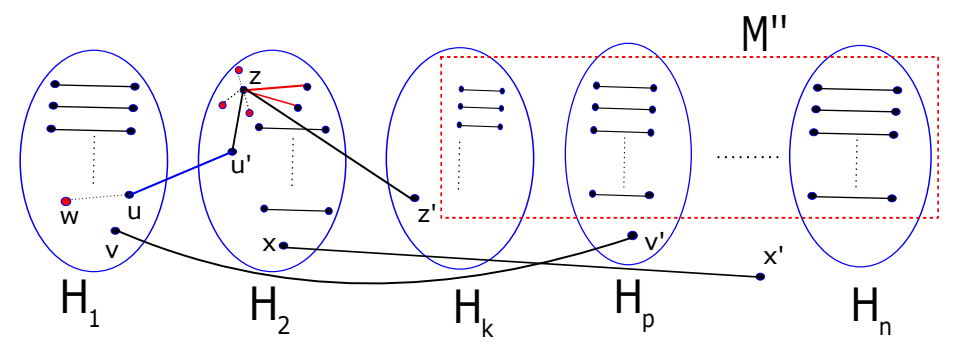

Figure 5: Case 2.2.2.1: $\left|F_{2}^{V}\right|$ is odd

Case 2.2.2.2. Assume that $F_{1}^{V}=\emptyset$. Then all the faults inside $H_{1}$ are edges. Then the vertex $u$ is incident to $(2 n-6)$ faulty edges in $H_{1}$. Deleting the vertex $u$ from $H_{1}$ includes the deletion of all those $(2 n-6)$ edges. Choose a vertex $v \in V\left(H_{1}-\{u\}\right)$ such that $H_{1}-\{u, v\}$ has no isolated vertices and $v$ has two outside neighbors in $A G_{n}-F$. By Theorem 2.4, there exists a perfect matching $M_{1}$ in $H_{1}-\{u, v\}$. Then $M_{1}$ is a perfect matching in $H_{1}-\left(F_{1} \cup\{u, v\}\right)$. The construction of the perfect or almost-perfect matching in $A G_{n}-F$ follows the same way as in Case 2.2.2.1.

Case 3. $\left|F_{1}\right|=2 n-6$ and $\left|F_{2}\right|=2 n-6$. Note that all the faults are in the subgraph induced by $V\left(H_{1}\right) \cup V\left(H_{2}\right)$.

Case 3.1. Assume that $H_{1}-F_{1}$ and $H_{2}-F_{2}$ have no isolated vertices. By the induction hypothesis, there exist perfect or almost-perfect matchings $M_{1}$ and $M_{2}$ in $H_{1}-F_{1}$ and $H_{2}-F_{2}$ respectively. If $M_{1}$ and/or $M_{2}$ are almost-perfect matchings, then at most two vertices will be missed by $M_{1} \cup M_{2}$. Let $x \in V\left(H_{1}-F_{1}\right)$ and $y \in V\left(H_{2}-F_{2}\right)$ be these vertices and let $x^{\prime} \in V\left(H_{p}-F_{p}\right)$ and $y^{\prime} \in V\left(H_{q}-F_{q}\right)$, where $p, q \neq 1,2$, be their corresponding outside neighbors. Since $\left|F_{i}\right|=0$ for $i \geq 3$, then $\left|F_{i} \cup\left\{x^{\prime}, y^{\prime}\right\}\right| \leq 2$ for $i \geq 3$, then we can use the construction of Case 1 to find a perfect or an almost-perfect matching $M^{\prime}$ in the subgraph induced by $\left(A G_{n}-F\right)-\left(V\left(H_{1}\right) \cup V\left(H_{2}\right) \cup\left\{x^{\prime}, y^{\prime}\right\}\right)$. Therefore $M_{1} \cup M_{2} \cup M^{\prime} \cup\left\{x x^{\prime}, y y^{\prime}\right\}$ is a perfect or an almost-perfect matching in $A G_{n}-F$.

Case 3.2. Assume that $H_{1}-F_{1}$ has an isolated vertex $u$, and $H_{2}-F_{2}$ has no isolated vertices. The vertex $u$ must be adjacent to a vertex $u^{\prime} \in V\left(H_{k}-F_{k}\right)$ for some $k \neq 1,2$ such that $u u^{\prime} \in E\left(A G_{n}-F\right)$. Since $\left|F_{1}\right|=2 n-6$ and $H_{1}-F_{1}$ has an isolated vertex, then we will consider two cases depending on whether $F_{1}$ is a strong matching preclusion set in $H_{1}$ or not.

Case 3.2.1. Assume that $F_{1}$ is not a matching preclusion set. By Proposition 2.1, $\left|F_{1}^{V}\right|$ is odd. Then $H_{1}-F_{1}$ has an almost-perfect matching $M_{1}$ missing the vertex $u$. By the induction hypothesis, $H_{2}-F_{2}$ has a perfect or an almost-perfect matching $M_{2}$ and $H_{i}-\left(F_{i} \cup\left\{u^{\prime}\right\}\right)$ has a perfect or an almost-perfect matching 
$M_{i}$ for $i \geq 3$. Using the same idea of Case 1 , we can find a perfect or an almost-perfect matching $M^{\prime}$ in the subgraph induced by $\left(A G_{n}-F\right)-$ $\left(V\left(H_{1}\right) \cup V\left(H_{2}\right) \cup\left\{u^{\prime}\right\}\right)$.

If $M_{2}$ is a perfect matching, then $M_{1} \cup M_{2} \cup M^{\prime} \cup\left\{u u^{\prime}\right\}$ is a perfect or an almost-perfect matching in $A G_{n}-F$.

If $M_{2}$ is an almost-perfect matching, then $M_{2}$ misses a vertex $x \in V\left(H_{2}-F\right)$ which has an outside neighbor $x^{\prime}$ in the subgraph induced by $\left(A G_{n}-F\right)-$ $\left(V\left(H_{1}\right) \cup V\left(H_{2}\right) \cup\left\{u^{\prime}\right\}\right)$. Note that we are assuming that $x^{\prime} \neq u^{\prime}$, since if $x^{\prime}=$ $u^{\prime}$, then by Proposition 2.1 we can find another almost-perfect matching in $H_{2}-F$ which misses a vertex other than $x$ and this vertex can not be adjacent to $u^{\prime}$. By the induction hypothesis, $H_{i}-\left(F_{i} \cup\left\{u^{\prime}, x^{\prime}\right\}\right)$ has a perfect or an almost-perfect matching $M_{i}^{\prime}$ for $i \geq 3$. Using the same idea of Case 1 , we can find a perfect or an almost-perfect matching $M^{\prime \prime}$ in the subgraph induced by $\left(A G_{n}-F\right)-\left(V\left(H_{1}\right) \cup V\left(H_{2}\right) \cup\left\{u^{\prime}, x^{\prime}\right\}\right)$. Thus the set $M_{1} \cup M_{2} \cup\left\{u u^{\prime}, x x^{\prime}\right\} \cup M^{\prime \prime}$ is a perfect or an almost-perfect matching in $A G_{n}-F$.

Case 3.2.2. Assume that $F_{1}$ is a matching preclusion set. Then by Proposition $2.1,\left|F_{1}^{V}\right|$ is even.

Case 3.2.2.1. Assume $F_{1}^{V} \neq \emptyset$. Let $w$ be a faulty vertex in $H_{1}$ and chose $x \in V\left(H_{1}-F_{1}\right)$ such that $H_{1}-\left(F_{1} \cup\{x\}\right)$ has no isolated vertices. Since all the faults are in the subgraph induced by $V\left(H_{1}\right) \cup V\left(H_{2}\right)$, then the vertex $x$ has at least one outside neighbor $x^{\prime} \in V\left(H_{k}-F_{k}\right)$ with $x x^{\prime} \in E\left(A G_{n}-F\right)$ for some $k \neq 1,2$. Let $F_{1}^{\prime}=\left(F_{1} \cup\{x\}\right)-\{w\}$, the subgraph $H_{1}-F_{1}^{\prime}$ has no isolated vertices and $\left|F_{1}^{\prime}\right|=2 n-6$ and $\left|F_{1}^{\prime V}\right|$ is even, then by Theorem 2.4 there exists a perfect matching $M_{1}$ in $H_{1}-F_{1}^{\prime}$. This matching $M_{1}$ includes the edge $u w$. By the induction hypothesis, $H_{2}-F$ has a perfect or an almost-perfect matching $M_{2}$.

Assume that $M_{2}$ is a perfect matching in $H_{2}-F_{2}$. By the induction hypothesis, $H_{i}-\left(F_{i} \cup\left\{u^{\prime}, x^{\prime}\right\}\right)$ has a perfect or an almost-perfect matching $M_{i}$ for $i \geq 3$, and since $\left|F_{i} \cup\left\{u^{\prime}, x^{\prime}\right\}\right| \leq 2 n-7$, then we proceed as in Case 1 to find a perfect or an almost-perfect matching $M^{\prime}$ in the subgraph induced by $\left(A G_{n}-F\right)-\left(V\left(H_{1}\right) \cup V\left(H_{2}\right) \cup\left\{u^{\prime}, x^{\prime}\right\}\right)$. Thus the set $\left(M_{1}-\right.$ $\{w v\}) \cup\left\{u u^{\prime}, x x^{\prime}\right\} \cup M^{\prime}$ is a perfect or an almost-perfect matching in $A G_{n}-F$.

Assume that $M_{2}$ is an almost-perfect matching, then $M_{2}$ misses a vertex $y \in V\left(H_{2}-F_{2}\right)$ which has an outside neighbor $y^{\prime}$ in the subgraph induced by $\left(A G_{n}-F\right)-\left(V\left(H_{1}\right) \cup V\left(H_{2}\right) \cup\left\{u^{\prime}, x^{\prime}\right\}\right)$. Again, we are assuming that $y^{\prime} \neq u^{\prime}$, since if $y^{\prime}=u^{\prime}$, then by Proposition 2.1 we can find another almost-perfect matching in $H_{2}-F_{2}$ which will miss a vertex other than $y$ and this vertex can not be adjacent to $u^{\prime}$. In addition, the choice of $x$ is not unique in $H_{1}-F_{1}$. By the induction hypothesis, $H_{i}-\left(F_{i} \cup\left\{u^{\prime}, x^{\prime}, y^{\prime}\right\}\right)$ has a perfect or an almost-perfect matching $M_{i}^{\prime}$ for $i \geq 3$. Using the same idea of Case 1, we can find a perfect or an almost-perfect matching $M^{\prime \prime}$ in the subgraph induced by $\left(A G_{n}-F\right)-\left(V\left(H_{1}\right) \cup V\left(H_{2}\right) \cup\left\{u^{\prime}, x^{\prime}, y^{\prime}\right\}\right)$. Thus the set $M_{1} \cup M_{2} \cup\left\{u u^{\prime}, x x^{\prime}, y y^{\prime}\right\} \cup M^{\prime \prime}$ is a perfect or an almost-perfect 
matching in $A G_{n}-F$.

Case 3.2.2.2. Assume $F_{1}^{V}=\emptyset$. Then $u$ is incident to $(2 n-6)$ faulty edges. Let $e$ be a faulty edge in $H_{1}$ and $u, w \in V\left(H_{1}-F_{1}\right)$ be its endpoints. The vertex $w$ must have an outside neighbor $w^{\prime} \in V\left(H_{p}-F_{p}\right)$ for some $p \neq 1,2$. Note that it is possible to have $p=k$. By the induction hypothesis, $H_{i}-\left(F_{i} \cup\left\{u^{\prime}, w^{\prime}\right\}\right)$ has a perfect or an almost-perfect matching $M_{i}$ for $i \geq 3$ and $H_{2}-F_{2}$ has a perfect or an almost-perfect matching $M_{2}$. We can now proceed as in the case above to find a perfect or an almost-perfect matching in $A G_{n}-F$.

Case 3.3. Assume that $H_{1}-F_{1}$ has an isolated vertex $u$, and $H_{2}-F_{2}$ has an isolated vertex $v$. Then $u$ must be incident to a cross edge that has its other endpoint in some $H_{k}-F_{k}$ where $k \neq 1,2$, and $v$ is incident to a cross edge that has its other endpoint in some $H_{j}-F_{j}$ where $j \neq 1,2$. Let $u u^{\prime}$ and $v v^{\prime}$ be those edges, so $u^{\prime} \in V\left(H_{k}-F_{k}\right)$ and $v^{\prime} \in V\left(H_{j}-F_{j}\right)$. It is possible to have $k=j$ and $u^{\prime}=v^{\prime}$. If $u^{\prime}=v^{\prime}$, then by Proposition 2.6, uv $\in E\left(A G_{n}-F\right)$, and we can include this edge in the matching to saturate $u$ and $v$.

Case 3.3.1. If $F_{1}$ and $F_{2}$ are not matching preclusion sets in $H_{1}$ and $H_{2}$ respectively. Then this case is similar to Case 3.2.1.

Case 3.3.2. If $F_{1}$ is a matching preclusion set in $H_{1}$ but $F_{2}$ is not a matching preclusion set in $\mathrm{H}_{2}$. Then this case is similar to Case 3.2.2.

Case 3.3.3. If $F_{1}$ and $F_{2}$ are matching preclusion sets in $H_{1}$ and $H_{2}$ respectively. Then $F_{1}$ and $F_{2}$ are basic strong matching preclusion sets in $H_{1}$ and $H_{2}$ respectively. By Proposition 2.1, $F_{1}$ and $F_{2}$ are trivial, so $\left|F_{1}^{V}\right|$ and $\left|F_{2}^{V}\right|$ are even. As in Case 3.2.2, we can always find a matching $M_{1}$ in $H_{1}-F_{1}$ missing the vertices $u, x \in V\left(H_{1}-F_{1}\right)$, and a matching $M_{2}$ in $H_{2}-F_{2}$ missing the vertices $v, y \in V\left(H_{2}-F_{2}\right)$. Since the choice of the vertices $x$ and $y$ is not unique, we can choose those vertices so that their outside neighbors do not lie in the same $H_{i}$. Let $u^{\prime}, x^{\prime}, v^{\prime}, y^{\prime}$ be the outside neighbors of $u, x, v, y$ respectively, such that $x^{\prime}$ and $y^{\prime}$ are in different $H_{i}$ 's. By the induction hypothesis, there exists a perfect or an almost-perfect matching $M_{i}$ in every $H_{i}-\left(F_{i} \cup\left\{u^{\prime}, x^{\prime}, v^{\prime}, y^{\prime}\right\}\right)$ for $i \geq 3$, and since $\left|F_{i} \cup\left\{u^{\prime}, x^{\prime}, v^{\prime}, y^{\prime}\right\}\right| \leq 2 n-7$ for $i \geq 3$, then we can proceed as in Case 1 and combine those matchings to get a perfect or an almost-perfect matching $M^{\prime}$ in the subgraph induced by $\left(A G_{n}-F\right)-\left(V\left(H_{1}\right) \cup V\left(H_{2}\right) \cup\right.$ $\left.\left\{u^{\prime}, x^{\prime}, v^{\prime}, y^{\prime}\right\}\right)$. Therefore, $M_{1} \cup M_{2} \cup M^{\prime} \cup\left\{u u^{\prime}, x x^{\prime}, v v^{\prime}, y y^{\prime}\right\}$ is a perfect or an almost-perfect matching in $A G_{n}-F$.

Case 4. $2 n-5 \leq\left|F_{1}\right| \leq 4 n-16$ for $n \geq 6$. When $n=5$, the compound inequality is not valid. So we must consider the cases when $n=5$ and $\left|F_{1}\right|=5$ or $\left|F_{1}\right|=6$. The case when $n=5$ and $\left|F_{1}\right|=5$ will be covered in Case 5. If $n=5$ and $\left|F_{1}\right|=6$ then, by Lemma 2.3, we can choose another decomposition via a different position so that $\left|F_{1}\right| \leq 5$.

Case 4.1. $H_{1}-F_{1}$ has no isolated vertices. By the induction hypothesis, $H_{1}-F_{1}$ contains a perfect or an almost-perfect matching $M_{1}$. We consider two cases depending on whether $M_{1}$ is a perfect or an almost-perfect matching. 
$M_{1}$ is a perfect matching. Since every $H_{i}-F_{i}$ contains no isolated vertices, then we can proceed as in Case 1 to find a perfect or an almost-perfect matching $M^{\prime}$ in the subgraph induced by $\left(A G_{n}-F\right)-V\left(H_{1}\right)$. Then the set $M_{1} \cup M^{\prime}$ is a perfect or an almost-perfect matching in $A G_{n}-F$.

$M_{1}$ is an almost-perfect matching. Let $u \in V\left(H_{1}-F_{1}\right)$ be the unsaturated vertex by $M_{1}$. If $u$ has an outside neighbor $u^{\prime}$ in some $H_{k}-F_{k}$ where $H_{k}-\left(F_{k} \cup\left\{u^{\prime}\right\}\right)$ contains no isolated vertices, then we can also proceed as in Case 1 by finding a perfect or an almost-perfect matching $M^{\prime}$ in the subgraph induced by $\left(A G_{n}-\right.$ $F)-\left(V\left(H_{1}\right) \cup\left\{u^{\prime}\right\}\right)$, so $M_{1} \cup M^{\prime} \cup\left\{u u^{\prime}\right\}$ is a perfect or an almost-perfect matching in $A G_{n}-F$. Assume that $u$ has no such outside neighbor. Since $H_{1}-F_{1}$ has no isolated vertices, then $u$ is adjacent to some vertex $v$ in $H_{1}-F_{1}$ and $v$ is saturated by $M_{1}$. Let $v w \in M_{1}$. The vertices $u$ and $w$ can have at most two common neighbors, so there are at least $4 n-14-\left|F_{1}\right|$ vertices in $H_{1}-F_{1}$ adjacent to either $u$ or $w$. Let $\overline{F_{1}}=F-F_{1}$; we know that $\left|\overline{F_{1}}\right|+\left|F_{1}\right|=4 n-12$, so there are at least $4 n-14-\left(4 n-12-\left|\overline{F_{1}}\right|\right)=\left|\overline{F_{1}}\right|+2$ vertices in $H_{1}-F_{1}$ adjacent to either $u$ or $w$. Then we can guarantee that $u$ or $w$ are adjacent to at least $\left\lfloor\frac{\overline{\mid F_{1}} \mid+2}{2}\right\rfloor$ vertices $x_{k}$ incident to edges $e_{k}=x_{k} y_{k} \in M_{1}$ for $k=1, \ldots,\left\lfloor\frac{\left|F_{1}\right|+2}{2}\right\rfloor$. We want to choose a vertex $y_{k}$ such that $y_{k}$ is not adjacent/incident to a fault outside $H_{1}$. Since each $y_{k}$ has two outside neighbors, then we have at least $\left|\overline{F_{1}}\right|+1$ choices for $y_{k}$; at most $\left|\overline{F_{1}}\right|$ can be at fault and no two outside neighbors can be in the same $H_{i}$. So we can find $y_{k}^{\prime} \in H_{k}-F$, where $y_{k} y_{k}^{\prime} \in E\left(A G_{n}-F\right)$, and satisfying the property $H_{k}-\left(F \cup\left\{y_{k}^{\prime}\right\}\right)$ has no isolated vertices. Thus we can use a similar construction to Case 1 to find a perfect or an almost-perfect matching $M^{\prime}$ in the subgraph induced by $\left(A G_{n}-F\right)-\left(V\left(H_{1}\right) \cup V\left(H_{2}\right) \cup\left\{y_{k}^{\prime}\right\}\right)$. If $w x_{k} \in E\left(H_{1}-F_{1}\right)$, then $M_{1}^{\prime}=M_{1} \cup\left\{w x_{k}, u v\right\}-\left\{v w, x_{k} y_{k}\right\}$ is an almost-perfect matching in $H_{1}-F_{1}$ missing $y_{k}$, then the set $M_{1}^{\prime} \cup M^{\prime} \cup\left\{y_{k} y_{k}^{\prime}\right\}$ is a perfect or an almost-perfect matching in $A G_{n}-F$. If $u x_{k} \in E\left(H_{1}-F\right)$, then $M_{1}^{\prime}=M_{1} \cup\left\{u x_{k}\right\}-\left\{x_{k} y_{k}\right\}$ is an almostperfect matching in $H_{1}-F$ missing $y_{k}$. Therefore, the set $M_{1}^{\prime} \cup M^{\prime} \cup\left\{y_{k} y_{k}^{\prime}\right\}$ is a perfect or an almost-perfect matching in $A G_{n}-F$.

Case 4.2. $H_{1}-F_{1}$ has an isolated vertex $u \in V\left(H_{1}-F_{1}\right)$. Let $N_{H_{1}}(u)$ be the set of neighbors of $u$ in $H_{1}$.

Case 4.2.1. $N_{H_{1}}(u) \cap F_{1}^{V} \neq \emptyset$. Let $w \in N_{H_{1}}(u) \cap F_{1}^{V}$. We consider the parity of $\left|F_{1}^{V}\right|$. Assume $\left|F_{1}^{V}\right|$ is odd. Let $F_{1}^{\prime}=F_{1}-\{w\}$, so $\left|F_{1}^{\prime}\right| \leq 4 n-17$ and $H_{1}-F_{1}^{\prime}$ has no isolated vertices, then by the induction hypothesis there exists a perfect matching $M_{1}$ in $H_{1}-F_{1}^{\prime}$. $M_{1}$ contains the edge $u w$. The vertex $u$ must have an outside neighbor $u^{\prime}$ in $A G_{n}-F$, or else $u$ becomes an isolated vertex in $A G_{n}-F$. By the induction hypothesis, there exists a perfect or an almost-perfect matching $M_{i}$ in $H_{i}-\left(F_{i} \cup\left\{u^{\prime}\right\}\right)$ for $i \geq 2$. Using the same idea as in Case 1 , we can find a perfect or an almost-perfect matching $M^{\prime}$ in the subgraph induced by $\left(A G_{n}-F\right)-\left(V\left(H_{1}\right) \cup\left\{u^{\prime}\right\}\right)$, then the set $\left(M_{1}-\{u w\}\right) \cup\left\{u u^{\prime}\right\} \cup M^{\prime}$ is a perfect or an almost-perfect matching in $A G_{n}-F$.

Assume $\left|F_{1}^{V}\right|$ is even. Let $F_{1}^{\prime}=F_{1}-\{w\}$, so $\left|F_{1}^{\prime}\right| \leq 4 n-17$ and $H_{1}-F_{1}^{\prime}$ has no isolated vertices. There exists a vertex $x \in V\left(H_{1}-F_{1}^{\prime}\right)$ such that $x$ has an 
outside neighbor $x^{\prime} \in V\left(H_{k}-F_{k}\right)$ with $x x^{\prime} \in E\left(A G_{n}-F\right)$ for some $k \neq 1$ and $H_{1}-\left(F_{1}^{\prime} \cup\{x\}\right)$ has no isolated vertices. By the induction hypothesis, there exists a perfect matching $M_{1}$ in $H_{1}-\left(F_{1}^{\prime} \cup\{x\}\right)$ that contains the edge $u w$, and there exists a perfect or an almost-perfect matching $M_{i}$ in $H_{i}-\left(F_{i} \cup\left\{u^{\prime}, x^{\prime}\right\}\right)$ for $i \geq 2$. Using the same idea as in Case 1, we can find a perfect or an almost-perfect matching $M^{\prime}$ in the subgraph induced by $\left(A G_{n}-F\right)-\left(V\left(H_{1}\right) \cup\left\{u^{\prime}, x^{\prime}\right\}\right)$, then the set $\left(M_{1}-\{u w\}\right) \cup\left\{u u^{\prime}, x x^{\prime}\right\} \cup M^{\prime}$ is a perfect or an almost-perfect matching in $A G_{n}-F$.

Case 4.2.2. $N_{H_{1}}(u) \cap F_{1}^{V}=\emptyset$. Then $u$ is incident to $(2 n-6)$ faulty edges in $H_{1}$. Let $f$ be one of those edges. We consider the parity of $\left|F_{1}^{V}\right|$. Assume $\left|F_{1}^{V}\right|$ is odd. Let $F_{1}^{\prime}=F_{1}-f$, where $f$ has endpoints $u$ and $v$ in $H_{1}-F_{1}$. Consider the set $F_{1}^{\prime \prime}=F_{1}^{\prime} \cup\{u\}$. $F_{1}^{\prime \prime}$ has an even number of vertices, of size less than or equal to $4 n-16$ and $H_{1}-F_{1}^{\prime \prime}$ has no isolated vertices. Then by the induction hypothesis, there exists a perfect matching $M_{1}$ in $H_{1}-F_{1}^{\prime \prime}$. The vertex $u$ must have an outside neighbor $u^{\prime} \in V\left(H_{k}-F_{k}\right)$ for some $k \neq 1$ such that $u u^{\prime} \in E\left(A G_{n}-F\right)$. By the induction hypothesis, there exists a perfect or an almost-perfect matching $M_{i}$ in $H_{i}-\left(F_{i} \cup\left\{u^{\prime}\right\}\right)$ for $i \geq 2$. We can assume that $\left|F_{k} \cup\left\{u^{\prime}\right\}\right| \leq 2 n-7$, since if not then $\left|F_{k}\right|=2 n-7$ and all the faults will be in the subgraph induced by $V\left(H_{1}\right) \cup V\left(H_{k}\right)$, so $u$ can have another outside neighbor in $H_{l}-F_{l}$ for some $l \neq 1, k$. Then $\left|F_{i} \cup\left\{u^{\prime}\right\}\right| \leq 2 n-7$ for $i \geq 2$ and hence we can apply the same idea as in Case 1 to construct a perfect or an almost-perfect matching $M^{\prime}$ in the subgraph induced by $\left(A G_{n}-F\right)-\left(V\left(H_{1}\right) \cup\left\{u^{\prime}\right\}\right)$. Then the set $M_{1} \cup\left\{u u^{\prime}\right\} \cup M^{\prime}$ is a perfect or an almost-perfect matching in $A G_{n}-F$.

Assume $\left|F_{1}^{V}\right|$ is even. Let $F_{1}^{\prime}=F_{1}-f$. We can choose the edge $f=u w$ such that $w$ has an outside neighbor $w^{\prime} \in V\left(H_{j}-F_{j}\right)$ for some $j \neq 1$ with $w w^{\prime} \in E\left(A G_{n}-F\right)$. The vertex $u$ must have an outside neighbor $u^{\prime} \in V\left(H_{k}-F_{k}\right)$ for some $k \neq 1$ such that $u u^{\prime} \in E\left(A G_{n}-F\right)$. Note that there are $(2 n-6)$ possible choices for $f$ and there are at most $(2 n-7)$ faults outside $H_{1}-F_{1}$, so we can always choose $w$ such that $w$ has two outside neighbors in $A G_{n}-F$. Then we can assume that $u^{\prime}$ and $w^{\prime}$ are in different $H_{i}$, so we can claim that $\left|F_{i} \cup\left\{w^{\prime}, u^{\prime}\right\}\right| \leq 2 n-7$. By the induction hypothesis, there exists a perfect or an almost-perfect matching $M_{i}$ in $H_{i}-\left(F_{i} \cup\left\{w^{\prime}, u^{\prime}\right\}\right)$ for $i \geq 2$. Using the same idea as in Case 1 , we can find a perfect or an almost-perfect matching $M^{\prime}$ in the subgraph induced by $\left(A G_{n}-F\right)-\left(V\left(H_{1}\right) \cup\left\{u^{\prime}, w^{\prime}\right\}\right)$. Therefore, the set $\left(M_{1}-u w\right) \cup\left\{u u^{\prime}, w w^{\prime}\right\} \cup M^{\prime}$ is a perfect or an almost-perfect matching in $A G_{n}-F$.

Case 5. $\left|F_{1}\right|=4 n-15$. Note that $H_{1}-F_{1}$ can have at most one isolated vertex. We will assume that $H_{1}-F_{1}$ has an isolated vertex $u \in V\left(H_{1}-F_{1}\right)$. The case when $H_{1}-F_{1}$ has no isolated vertices will be treated the same. Under this condition, there are three faults left outside $H_{1}$. Since $A G_{n}-F$ has no isolated vertices then there exists an outside neighbor $u^{\prime} \in V\left(H_{p}-F_{p}\right)$ for some $p \neq 1$ with $u u^{\prime} \in E\left(A G_{n}-F\right)$.

Case 5.1. $N_{H_{1}}(u) \cap F_{1}^{V}=\emptyset$. Then $u$ is incident to $(2 n-6)$ edges. Let $f=u v$ be a faulty edge in $H_{1}$ such that $v$ has two outside neighbors in $A G_{n}-F$. Let $v^{\prime} \in V\left(H_{k}-F\right)$, for some $k \neq 1, p$, be an outside neighbor of $v$. Since $\left|F_{1}-f\right|=4 n-16$ and 
$H_{1}-\left(F_{1}-f\right)$ has no isolated vertices, then by the induction hypothesis there exists a perfect or an almost-perfect matching $M_{1}$ in $H_{1}-\left(F_{1}-f\right)$.

Assume that $f \notin M_{1}$. Then $M_{1}$ is a perfect or an almost-perfect matching in $H_{1}-F_{1}$. By the induction hypothesis, there exists a perfect or an almost-perfect matching $M_{i}$ in $H_{i}-F_{i}$. We can combine these matchings to get a perfect or an almost-perfect matching $M$ in $\left(A G_{n}-F\right)$.

Assume that $f \in M_{1}$. There are three faults outside $H_{1}$, we can claim that $\left|F_{i} \cup\left\{u^{\prime}\right\}\right| \leq 3$ for every $i \geq 2$, because if not then $u^{\prime}$ is in the same $H_{i}$ along with the other faults and this guarantees the existence of another outside neighbor of $u$ in $A G_{n}-F$. Moreover, $v$ has two outside neighbors in $A G_{n}-F$ so we can claim that $\left|F_{i} \cup\left\{u^{\prime}, v^{\prime}\right\}\right| \leq 2 n-7$ for $i \geq 2$. Now as in Case 1 we can find a perfect or an almost-perfect matching $M^{\prime}$ in the subgraph induced by $\left(A G_{n}-F\right)-\left(V\left(H_{1}\right) \cup\left\{u^{\prime}, v^{\prime}\right\}\right)$. Therefore $M_{1} \cup M^{\prime} \cup\left\{u u^{\prime}, v v^{\prime}\right\}$ is a perfect or an almost-perfect matching in $A G_{n}-F$.

Case 5.2. $N_{H_{1}}(u) \cap F_{1}^{V} \neq \emptyset$. Let $w \in N_{H_{1}}(u) \cap F_{1}^{V}$. By the induction hypothesis, there exists a perfect or an almost-perfect matching $M_{1}$ in $H_{1}-\left(F_{1}-\{w\}\right)$. We consider the parity of $\left|F_{1}^{V}\right|$.

$\left|F_{1}^{V}\right|$ is odd. Then $M_{1}$ is a perfect matching containing the edge $u w$. As in the previous case, we can claim that $\left|F_{i} \cup\left\{u^{\prime}\right\}\right| \leq 3$ for every $i \geq 2$, so by the induction hypothesis there exists a perfect or an almost-perfect matching $M_{i}$ in $H_{i}-\left(F_{i} \cup\left\{u^{\prime}\right\}\right)$ for $i \geq 2$. We combine all the $M_{i}$ 's to get a perfect or an almostperfect matching $M^{\prime}$ in the subgraph induced by $\left(A G_{n}-F\right)-\left(V\left(H_{1}\right) \cup\left\{u^{\prime}\right\}\right)$. Therefore $\left(M_{1}-\{u w\}\right) \cup M^{\prime} \cup\left\{u u^{\prime}\right\}$ is a perfect or an almost-perfect matching in $A G_{n}-F$.

$\left|F_{1}^{V}\right|$ is even. Then $M_{1}$ is an almost-perfect matching in $H_{1}-\left(F_{1}-\{w\}\right)$. Let $M_{1}^{\prime}=M \cap E\left(H_{1}-F\right) . \quad M_{1}^{\prime}$ is a matching in $H_{1}-F$ that misses two vertices, one of them is $u$ and some other vertex $x$. Note that $M_{1}^{\prime}$ is an almost-perfect matching in the subgraph induced by $H_{1}-\left(F_{1} \cup\{u\}\right)$, so we claim that $x$ has outside neighbor(s) in $A G_{n}-F$, because if not then by Proposition 2.1 we can find another almost-perfect matching in $H_{1}-\left(F_{1} \cup\{u\}\right)$ missing a vertex other than $x$. Let $x^{\prime}$ be the outside neighbor of $x$ in $A G_{n}-F$. As in the previous case we can claim that $\left|F_{i} \cup\left\{x^{\prime}, u^{\prime}\right\}\right| \leq 2 n-7$ for $i \geq 2$. By the induction hypothesis there exists a perfect or an almost-perfect matching $M_{i}$ in $H_{i}-\left(F_{i} \cup\left\{x^{\prime}, u^{\prime}\right\}\right)$ for $i \geq 2$. We combine all the $M_{i}$ 's to get a perfect or an almost-perfect matching $M^{\prime}$ in the subgraph induced by $\left(A G_{n}-F\right)-\left(V\left(H_{1}\right) \cup\left\{x^{\prime}, u^{\prime}\right\}\right)$. Therefore $M_{1}^{\prime} \cup M^{\prime} \cup\left\{u u^{\prime}, x x^{\prime}\right\}$ is a perfect or an almost-perfect matching in $A G_{n}-F$.

\section{Conclusion}

In this paper, we have studied the strong matching preclusion problem of the alternating group graph under the condition that no isolated vertex is created as a result of faulty edges and/or vertices. We proved that the conditional strong matching preclusion number of $A G_{n}$ 
is $4 n-11$. Classifying all types of optimal conditional strong matching preclusion sets of $A G_{n}$ is left as a future research.

\section{References}

[1] R. Ledermann. Introduction to the Theory of Finite Groups. Olivier and Boyd, London, 1949.

[2] V. Kumar, A. Grama, A. Gupta, and G. Karypis. Introduction to Parallel Computing: Design and Analysis of Algorithms. Benjamin/Cummings, 1994.

[3] R. C. Brigham, F. Harary, E.E. Violin, and J. Yellen. Perfect matching preclusion, Congressus Numerantium, 174: 185-192, 2005.

[4] P. Bonneville, E. Cheng, and J. Renzi. Strong matching preclusion for the alternating group graphs and split-stars. Journal of Interconnection Networks, 12(4): 277-298, 2011.

[5] J.S. Jwo, S. Lakshmivarahan, and S.K. Dhall. A new class of interconnection networks based on the alternating group. Networks, 23: 315-326, 1993.

[6] N. Biggs. Algebraic Graph Theory. Cambridge University Press, 1979.

[7] J.-H. Park and I. Ihm. Strong matching preclusion. Theoretical Computer Science, 412: 6409-6419, 2011.

[8] J.-H. Park and I. Ihm. Strong matching preclusion under the conditional fault model. Discrete Applied Mathematics, 161: 1093-1105, 2013.

[9] E. Cheng, M.J. Lipman, and H.A. Park. Super connectivity of star graphs, alternating group graphs and split-stars. Ars Combinatoria, 59: 107-116, 2001.

[10] E. Cheng, L. Lesniak, M.J. Lipman, and L. Lipták. Conditional matching preclusion sets. Information Sciences, 179(8): 1092-1101, 2009.

[11] D.B. West. Introduction to Graph Theory. Prentice Hall, 1996.

[12] Z. Zhang, W. Xiong, and W. Yang. A kind of conditional fault tolerance of alternating group graphs. Information Processing Letters, 110: 998-1002, 2010. 